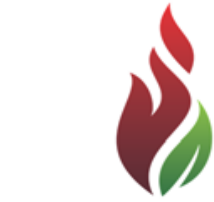

SUSTENERE

Publishing Corporation

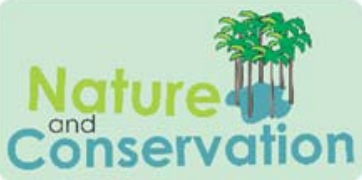

Journals Homepage:

www.sustenere.co/journals

\section{CARACTERIZACIÓN FLORÍSTICA DE LA RESERVA NATURAL MILITAR ESTANCIA LA CALERA}

\section{RESUMEN}

Las Sierras de Córdoba experimentan en la actualidad transformaciones causadas por el avance inmobiliario, incendios, tala, sobrepastoreo e invasión de especies exóticas. Estas transformaciones condicionan el estado de conservación de la flora nativa. La Reserva Militar Natural La Calera, ubicada en las Sierras de Córdoba, ha mantenido gran parte de su estructura vegetal original como consecuencia de las restricciones al ingreso debido al uso militar. El presente trabajo muestra los resultados de las actividades de diagnóstico florístico llevados a cabo en esta área de 14 mil hectáreas durante 2012 y 2013. Se realizó una clasificación ISO Cluster como base para los relevamientos florísticos realizados a través del Método Fitosociológico de Braun Blanquet. Se calcularon los Índices de Biodiversidad de Shannon-Weaver y se determinaron las especies características de cada ambiente, basados en los valores de abundancia y dominancia. A través de un análisis discriminante se determinaron las clases de vegetación, las cuales permitieron definir las mismas en una clasificación supervisada. Fueron identificadas 230 especies, de las cuales el 8,3\% son exóticas, valor inferior a la proporción de exóticas encontradas para el total de las Sierras de Córdoba. Estas especies exóticas se restringen a zonas riparias. El 17,0\% del total de especies relevado son endémicas de la ecorregión Chaqueña, lo que hace de esta Reserva un lugar de alto valor de conservación. Seis comunidades vegetales fueron identificadas, representadas en cuatro clases de vegetación en la clasificación supervisada.

PALABRA CLAVE: Sierras de Córdoba; Especies Nativas; Especies Exóticas; Especies Endémicas; Comunidades Vegetales.

\section{CARAVTERIZAÇÃO FLORÍSTICA DA RESERVA NATURAL MILITAR ESTANCIA LA CALERA}

\section{RESUMO}

As serras de Cordoba vem experimentando atualmente transformações provocadas pelo progresso de imóveis, incêndios, exploração madeireira, o sobrepastoreio e a invasão de espécies exóticas. Essas transformações determinão a condição da flora nativa. A Reserva Natural Militar Estancia La Calera, localizada nas serras de Córdoba, tem mantido grande parte da sua estrutura da vegetação original devido a restrições de entrada devido a uso militar. Este artigo apresenta os resultados das atividades de diagnóstico florístico realizados nesta área de 14 mil hectares em 2012 e 2013. Foi realizada uma classificação ISSO Cluster como base para levantamentos florísticos realizados através do método fitossociológico de Braun Blanquet. Índices de biodiversidade de espécies de Shannon-Weaver foram calculados e as características de cada ambiente com base nos valores de abundância e dominância foram determinadas. Através da análise discriminante classes de vegetação foram determinadas, o que permitiu definir-los em uma classificação supervisionada. Foram identificadas 230 espécies, das quais 8,3\% são exóticas, menos do que a proporção de estrangeiro encontrado para o valor total de Córdoba Sierras. Estas espécies exóticas são restritas a áreas ribeirinhas. $17,0 \%$ de todas as espécies são endêmicas aliviados com a ecorregião Chaco, tornando esta reserva um lugar de alto valor de conservação. Foram identificados seis comunidades da planta, representada em quatro tipos de vegetação na classificação supervisionada.

PALVRA-CHAVE: Sierras de Córdoba; Espécies Nativas; Espécies Exóticas; Espécies Endêmicas; Comunidades de Planta.
Nature and Conservation, Aquidabã, v.7, n.1, Nov, Dez 2013, Jan, Fev, Mar, Abr, Mai, Jun, Jul, Ago, Set, Out 2014.

\section{ISSN 2318-2881}

\section{SECTION: Articles}

TOPIC: Conservação da Natureza

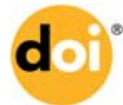

DOI: 10.6008/SPC2318-2881.2014.001.0001

Marcos Sebastián Karlin

Universidad Nacional de Córdoba, Argentina http://lattes.cnpq.br/6092043513312058

mkarlin@agro.unc.edu.ar

Cristian Schneider

Universidad Católica de Córdoba, Argentina http://lattes.cnpq.br/2229300213305426

cristian schneider@hotmail.com

Silvana Rufini

Universidad Nacional de Córdoba, Argentina silyruf@gmail.com

Javier Bernasconi

Universidad Nacional de Córdoba, Argentina http://lattes.cnpq.br/6730789827954920 aiijavier@yahoo.com

Ricardo Accietto

Universidad Nacional de Córdoba, Argentina accietto@agro.unc.edu.ar

Ulf Karlin

Universidad Nacional de Córdoba, Argentina http://lattes.cnpq.br/9672121343950501 ukarlin@gmail.com

Yamila Ferreyra

Universidad Nacional de Córdoba, Argentina yam.ferreyra@gmail.com

Received: 06/03/2014

Approved: 09/08/2014

Reviewed anonymously in the process of blind peer

Referencing this:

KARLIN, M. S.; SCHNEIDER, C.; RUFINI, S.; BERNASCONI, J.; ACCIETTO, R.; KARLIN, U.; FERREYRA, Y. Caracterización florística de la Reserva Natural Militar 'Estancia La Calera'. Nature and Conservation, Aquidabã, v.7, n.1, p.6-18, 2014. DOI: http://dx.doi.org/10.6008/SPC2318 2881.2014.001.0001 


\section{INTRODUCCIÓN}

En el centro de la Argentina se desarrollan las Sierras de Córdoba ubicadas en el Distrito Chaqueño Serrano (CABRERA, 1976). La vegetación característica de este Distrito es un bosque xerófilo a subxerófilo dominado, según Giorgis et al. (2011), por Schinopsis marginata Engl. y Lithraea molleoides (Vell.) Engl. La composición florística de estos bosques cambia con las características edáficas, topográficas y la historia de disturbio (CANTERO et al., 2011; GURVICH et al., 2005; SUÁREZ et al., 1997; CABIDO et al., 1991). Esto determina que el ecosistema sea altamente heterogéneo y que haya variaciones importantes en la composición de especies en toda su extensión (GIORGIS et al., 2011). En el piedemonte se encuentra el ecotono con la Provincia Fitogeográfica del Espinal, Distrito Cordubense (LEWIS et al., 1973), dominado por Aspidosperma quebracho-blanco Schltdl. y varias especies del género Prosopis, área altamente fragmentada por el avance de la frontera agropecuaria.

Los disturbios por fuego que ocurren cíclicamente todos los años en mayor o menor intensidad de acuerdo a la acumulación de combustible, han convertido parte de la vegetación original en arbustales y pastizales, los cuales son particularmente susceptibles a nuevos incendios, debido a la presencia de biomasa de fácil ignición en época seca. Las Sierras de Córdoba están experimentando en la actualidad grandes transformaciones, causadas principalmente por el avance de la red de urbanización, los incendios, la tala, el sobrepastoreo y la invasión de especies exóticas. Estas transformaciones condicionan fuertemente el establecimiento y supervivencia de algunas de las especies nativas (PALACIOS et al., 2010), poniendo a numerosas especies serranas en alto riesgo de extinción local.

En relación a esta problemática ambiental, la Reserva Natural Militar 'Estancia La Calera', ubicada entre dos grandes centros urbanos, como son Córdoba y Villa Carlos Paz, ha mantenido gran parte de su estructura vegetal original como consecuencia de las restricciones al ingreso debido a su uso militar. Esta Reserva constituye, por lo tanto, un lugar de referencia para evaluar el estado de la flora serrana y del Espinal de otros sitios de la región. Esta área natural fue expropiada por el Estado Nacional en el año 1943 para el uso y administración del Ejército Argentino, y destinada a prácticas militares y arrendamientos agrícolas y ganaderos. Los lotes agrícolas están ubicados en la zona este de la reserva, correspondiente a la región del Espinal, y en la actualidad se encuentra en franca reducción de su superficie debido al abandono progresivo de la actividad. Los lotes ganaderos se ubican en las zonas serranas, aunque las cargas animales son menores a la receptividad ganadera de los potreros. Este uso ininterrumpido durante 69 años ha permitido una 'clausura' espontánea que tuvo ciertos efectos de conservación sin modificaciones fundamentales de especies características del Chaco Serrano. Sin embargo, el área ecotonal con el Espinal dentro de la Reserva sufrió las consecuencias del avance de la agricultura, dejando sólo pequeños relictos de bosque nativo que es necesario recuperar. 
El instrumento legal que posibilitó la inclusión del área como espacio de conservación fue el Convenio Marco de Cooperación 100/07 entre el Ministerio de Defensa y la Administración de Parques Nacionales (APN), y el Protocolo Adicional N5 entre el Ejército Argentino y la APN, que a través de la figura de Espacios Naturales de Interés para la Conservación de la Biodiversidad (ENIC) como política de estado, amplía las áreas de conservación del patrimonio natural. En este contexto se desarrolla el proyecto 'El manejo como herramienta de recuperación de cuenca' para la Reserva Natural Militar 'Estancia La Calera' el cual implica, entre otras, la realización de actividades de diagnóstico que permitan definir una línea base a partir del cual se propondrán prácticas de manejo para la futura reglamentación de uso y conservación de los recursos naturales de la cuenca.

El objetivo de este trabajo es el de caracterizar la flora de la Reserva Natural Militar 'Estancia La Calera', teniendo en cuenta sus rasgos sobresalientes, tales como endemismos y presencia de especies exóticas, identificando zonas homogéneas para el manejo y conservación. El presente trabajo muestra los resultados de las actividades de diagnóstico florístico llevados a cabo desde el 21 de junio del 2012 al 6 de febrero de 2013 en la Reserva Natural Militar "Estancia La Calera", un área que no ha sido relevada a campo anteriormente, al menos desde el punto de vista florístico, que representa un sitio representativo de la flora nativa original de las Sierras Chicas de Córdoba, espacio dedicado a la conservación del sector oriental del Chaco Serrano y único representativo del Distrito Cordubense del Espinal.

\section{MATERIAL Y MÉTODOS}

\section{Ubicación del Área de Estúdio}

El área denominada históricamente como "Campos del III Cuerpo de Ejército" se encuentra al oeste de la ciudad de Córdoba y comprende aproximadamente unas 14 mil hectáreas. La Reserva Natural Militar, incluida en los predios de estos Campos, posee como límites físicos la Ruta Nacional $\mathrm{N}^{\circ} 20$ hacia el sur, la Ruta Provincial $\mathrm{N}^{\circ} 73$ hacia el oeste y el norte, y parte del anillo de circunvalación de la Ciudad de Córdoba hacia el este (Figura 1). Las coordenadas geográficas

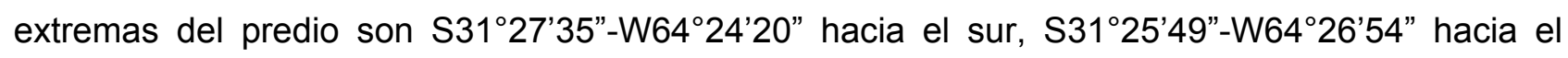

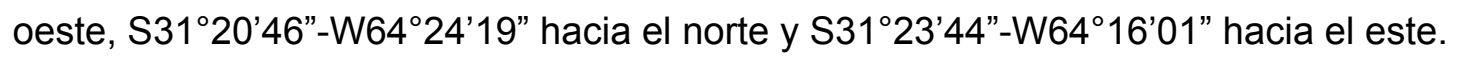

La precipitación media anual es de $800 \mathrm{~mm}$ anuales, con distribución marcadamente estival $(640 \mathrm{~mm})$. La temperatura media anual es de $16^{\circ} \mathrm{C}$, con una máxima media anual de $24^{\circ} \mathrm{C}$ y una mínima media anual de $10,1^{\circ} \mathrm{C}$. Las máximas absolutas alcanzan los $44^{\circ} \mathrm{C}$ y las mínimas absolutas de $-10^{\circ} \mathrm{C}$ (DALMASSO et al., 1997). Los vientos predominantes son del noreste y sursureste, de los cuales los primeros son cálidos y secos y los segundos son frescos y húmedos (CAPITANELLI, 1979). 


\section{Fase Exploratoria}

Una imagen satelital RapidEye de alta resolución espacial ( $5 \mathrm{~m}$ por pixel) de noviembre del 2010, correspondiente al área de estudio, fue sometida a un proceso de clasificación no supervisada sobre la base de sus 5 bandas espectrales, mediante el uso del módulo de clasificación de imágenes del software ArcGIS ${ }^{T M} 10$ (ESRI®) con el algoritmo ISO Cluster en base a 10 clases. Para diferenciar las unidades de cobertura de vegetación nativa de otras clases de cobertura del suelo, se utilizó un primer criterio de categorización de "vegetación" y "no vegetación" (DI GREGORIO et al., 2005). Para incluir la heterogeneidad regional de la flora en las unidades de cobertura de "vegetación", las mismas fueron determinadas en función de la fisonomía de los ambientes (MALDONADO et al., 2004; ETIENNE et al., 1982), de las descripciones fisonómicas y fitosociológicas publicadas anteriormente por diversos autores para el área de las Sierras de Córdoba (MORELLO et al., 1968; LUTI et al., 1979; ZAK et al., 2002; GAVIER et al., 2004).

\section{Relevamiento de La Vegetación}

Se realizó un inventario florístico mediante el Método Fitosociológico de Braun Blanquet (BRAUN BLANQUET, 1979; MUELLER-DOMBOIS et al., 1974), trabajando en las unidades homogéneas de vegetación definidas previamente (se descartaron las unidades de 'no vegetación' y 'agricultural'). Se definieron 44 sitios de reconocimiento de especies de una superficie de aproximadamente 1 ha cada uno. Este número responde a lo citado por Roig (1973) quien sugiere un número mínimo de relevamientos por este método representando al menos el $80 \%$ de las especies totales encontradas en el área de estudio total. En los sitios seleccionados, se estimaron los valores de abundancia-dominancia (BRAUN BLANQUET, 1979), los cuales se transformaron posteriormente en valores de porcentaje de cobertura basado en el punto medio de cada valor de la escala de abundancia-cobertura (WIKUM et al., 1978):

\footnotetext{
Individuos raros o poco frecuentes con cobertura insignificante: 0,5\%; Individuos abundantes, pero con cobertura insignificante: 2,5\%; Individuos en número variable, pero con cobertura de hasta $1 / 4$ de la superficie total: $15,0 \%$; Individuos en número variable, con cobertura entre $1 / 4$ a $1 / 2$ de la superficie total: $37,5 \%$; Individuos en número variable, con cobertura entre $1 / 2$ a $3 / 4$ de la superficie total: $62,5 \%$; Individuos en número variable, con cobertura superior a $3 / 4$ de la superficie total: $87,5 \%$.
}

Con esta escala mixta se elaboró una tabla fitosociológica sintética modal que resume las condiciones de cada uno de los ambientes relevados. Las especies indicadas en esta tabla corresponden a aquellas que poseen valor mayor a $2(15,0 \%$ de cobertura media) en relación a la escala de abundancia-cobertura en por lo menos uno de los ambientes identificados. 
Para las especies exóticas se calculó el área basal total considerando el diámetro a la altura de la base de individuos encontrados sobre transectas de $50 \times 6 \mathrm{~m}$ ubicadas al azar (GAILLARD DE BENITEZ et al., 2011) en 14 de los 44 sitios relevados.

\section{Análisis de Datos}

Con los valores de abundancia-cobertura se efectuó un análisis discriminante (Distancia Euclídea) utilizando como variables los valores medios de abundancia-cobertura de las 201 especies encontradas en los 44 sitios de muestreo, y como criterio de agrupamiento las comunidades vegetales identificadas. Con los valores de frecuencia se definieron las especies características mediante ANAVA, con el test LSD Fisher, con una probabilidad menor a 0,05 y 38 grados de libertad.

Se calcularon los Índices de Biodiversidad de Shannon-Weaver basados en los valores de abundancia y dominancia:

$$
H=-\sum_{i=1}^{n} p_{i} \ln p_{i}
$$

donde $p_{i}=N_{i} / \sum_{i=1}^{n} N_{i}$, n es el número de especies de cada comunidad vegetal y $N_{i}$ es el tamaño de cada población definida por la frecuencia relativa o cobertura de acuerdo a Wikum y Shanholtzer (1978).

Para todos los análisis estadísticos se utilizó el software estadístico Infostat (DI RIENZO et al., 2012). Las características anatómicas de las especies encontradas fueron comparadas con las descripciones efectuadas por Demaio et al. (2002) para árboles, por Sércic y Cocucci (2010) para herbáceas y arbustivas, por Volkmann y von Müller (1999) y Volkmann (2005) para enredaderas, por Trevisson y Demaio (2006) y Kiesling y Ferrari (2005) para cactáceas, y por Arana y Bianco (2011) para helechos y licófitas. Los nombres científicos fueron actualizados de acuerdo al criterio del Instituto de Botánica Darwinion, a marzo de 2013.

\section{Clasificación Supervisada}

En base a los relevamientos de vegetación realizados en cada una de las unidades de coberturas del suelo obtenidas en la clasificación ISO Cluster, se procedió a comprobar la asignación de las diferentes fisonomías a estas unidades establecidas por el software clasificador. Se efectuaron reasignaciones en algunos casos de acuerdo a las fisonomías identificadas a campo, y en otros casos cuando fue necesario unificar unidades similares, para obtener una clasificación supervisada final, a posteriori, de 7 clases diferentes como unidades homogéneas. La clasificación supervisada fue estimada usando los algoritmos de máxima verosimilitud y vecino más próximo en ArcGIS ${ }^{\text {TM }} 10$ (Proyección Tranverse Mercator Faja 4 - Datum WGS84). 


\section{RESULTADOS}

\section{Especies vegetales}

Fueron identificadas en el área de estudio un total de 230 especies, de las cuales 201 $(87,4 \%)$ fueron encontradas e identificadas en los 44 sitios. Diez especies fueron reconocidas sólo a nivel de género. Fueron encontradas 19 especies exóticas, de las cuales Morus alba L. y Ligustrum lucidum W.T. Aiton son las de mayor abundancia (hasta 37,5 y 15,0\% de cobertura del suelo respectivamente en los 44 sitios de muestreo de abundancia-cobertura) y área basal (valores máximos de $9,75 \mathrm{~m}^{2} /$ ha para $M$. alba; L. lucidum sin individuos adultos dentro de los 14 sitios de muestreo forestal). Estas se encuentran asociadas a cursos permanentes de agua y crecen en bosques densos asociados generalmente a Sebastiania commersoniana (Baill.) L.B. Sm. y Downs, también de gran abundancia y cobertura, en lo que hemos definido como 'blanquillales'.

Es de destacar la presencia de la especie Maclura pomifera (Raf.) Schneid. (un sólo individuo encontrado hasta el momento), especie que no se encuentra citada en la base de datos de Darwinion (2013). Fueron también identificadas Dolichandra unguis-cati (L.) L.G. Lohmann, Manihot grahamii Hook. (también asociado a los blanquillales, con valores máximos de cobertura de $25 \%$ y máxima área basal de $4,52 \mathrm{~m}^{2} / \mathrm{ha}$ ) y Guadua $\mathrm{sp}$., las cuales son especies nativas de Argentina pero no corresponden originalmente a esta ecorregión. Se encontraron 39 especies endémicas, según el status establecido por el Instituto Darwinion (a marzo del año 2013), algunas del centro norte del país y otras de las Sierras de Córdoba y San Luis. La mayoría de las especies endémicas serranas son cactáceas: Acanthocalycium spiniflorum (K. Schum.) Backeb., Echinopsis aurea var. aurea Britton y Rose, Gymnocalycium bruchii (Speg.) Hosseus, G. castellanosii Backeb. y G. monvillei (Lem.) Britton y Rose, Trichocereus candicans (Gillies ex Salm-Dyck) Britton y Rose y T. lamprochlorus (Lem.) Britton y Rose. Estas le dan valor a la Reserva como ámbito de protección de especies.

\section{Comunidades Vegetales y Clasificación de Unidades de Cobertura del Suelo}

De acuerdo a la Tabla Fitosociológica Sintética Modal (Tabla 1) y la Tabla de Especies Características (Tabla 2), se definieron seis comunidades vegetales de acuerdo a los valores de dominancia relevados, y a partir de esto se obtuvo la clasificación supervisada definitiva. El análisis discriminante (DI RIENZO et al., 2012) no arrojó errores de clasificación (tasa de error aparente $=0 \% ; \alpha=95 \%$ ). 
Tabla 1: Cuadro fitosociológico sintético modal en base a los censos realizados. $r$ : Renovales, $+: 0,5 \%, 1$ : 2,5\%, 2: $15 \%, 3: 37,5 \%, 4: 62,5 \%, 5: 87,5 \%$. Remarcado, las especies más relevantes de cada comunidad.

\begin{tabular}{|c|c|c|c|c|c|c|}
\hline $\begin{array}{l}\text { Sitios } \\
\text { Elevación (m.s.n.m.) } \\
\text { Exposición }\end{array}$ & $\begin{array}{l}\text { Quebrachal } \\
\text { 557 } \\
\text { SE }\end{array}$ & $\begin{array}{l}\text { Pastizal } \\
567 \\
\text { NE } \\
\text { S31 } 2613\end{array}$ & $\begin{array}{c}\text { Espinillal } \\
680 \\
N \\
\text { s } \\
312457\end{array}$ & $\begin{array}{l}\text { Blanquillal } \\
607 \\
\mathrm{~S} \\
5312220\end{array}$ & $\begin{array}{c}\text { Mollal } \\
638 \\
\text { SE } \\
S 312206\end{array}$ & $\begin{array}{l}\text { Horcal } \\
611 \\
E \\
S 312240\end{array}$ \\
\hline Coordenadas geográficas & $\begin{array}{r}\text { S312602 } \\
\text { W64 } 1953\end{array}$ & $\begin{array}{l}\text { S312613 } \\
\text { W64 2017 }\end{array}$ & $\begin{array}{l}\text { S31 24 57 } \\
\text { W64 23 55 }\end{array}$ & $\begin{array}{l}\text { S31 } 2220 \\
\text { W64 23 14 }\end{array}$ & $\begin{array}{l}\text { S3122 } 06 \\
\text { W64 2328 }\end{array}$ & $\begin{array}{l}S 312240 \\
\text { W64 22 } 58\end{array}$ \\
\hline 1. Schinus fasciculatus & 3 & + & & & & \\
\hline 2. Setaria parviflora & 2 & 1 & + & & & \\
\hline 3. Jodina rhombifolia & 2 & & & & + & \\
\hline 4. Aspidosperma quebracho-blanco & 3 & + & & & & \\
\hline 5. Cestrum parqui & 3 & 1 & & & 1 & \\
\hline 6. Celtis ehrenbergiana & 3 & 1 & & & 1 & + \\
\hline 7. Acacia praecox & 2 & & & $\mathrm{r}$ & & + \\
\hline 8. Acacia aroma & & 1 & 3 & & & \\
\hline 9. Acacia caven & + & 1 & 1 & & & \\
\hline 10. Jarava ichu var. ichu & & 2 & 2 & & & \\
\hline 11. Aloysia gratissima & 1 & 3 & 1 & & 1 & 1 \\
\hline $\begin{array}{l}\text { 12. Setaria lachnea } \\
\text { 13. Sorghum halepensis }\end{array}$ & 1 & $\begin{array}{l}2 \\
2\end{array}$ & & & & \\
\hline $\begin{array}{l}\text { 14. Bidens subalternans var. } \\
\text { subalternans }\end{array}$ & & 1 & & 2 & & \\
\hline 15. Croton hirtus & 1 & & + & 1 & 2 & \\
\hline 16. Croton lachnostachyus & + & & & 4 & 3 & \\
\hline 17. Sebastiania commersoniana & & & & 5 & 1 & 1 \\
\hline 18. Lithraea molleoides & & & & & 3 & \\
\hline 19. Abutilon grandifolium & & 1 & & & 2 & \\
\hline 20. Mandevilla pentlandiana & & & & 1 & 2 & \\
\hline 21. Schinopsis marginata & & & & 1 & & 3 \\
\hline 22. Flourensia oolepis & & & & & & 2 \\
\hline 23. Sida argentina & & & + & & & 1 \\
\hline 24. Amelichloa brachychaeta & & & 1 & & & \\
\hline 25. Salpichroa origanifolia & & + & & & 1 & \\
\hline
\end{tabular}

Tabla 2: Especies características.

\begin{tabular}{|c|c|c|c|c|c|c|}
\hline Especie* $^{*}$ & Quebrachal $(n=3)$ & Espinillal (n=11) & Pastizal $(n=14)$ & Blanquillal $(n=9)$ & Mollal $(n=3)$ & Horcal $(n=4)$ \\
\hline Abu gra & 0 & 0,01 & 0,01 & 0,01 & 0,06 & 0 \\
\hline Аbu раu & 0,03 & 0,01 & 0 & 0 & 0 & 0 \\
\hline Aca aro & 0 & 0,10 & 0,02 & 0 & 0 & 0 \\
\hline Aca cav & 0 & 0,18 & 0,06 & 0 & 0,01 & 0 \\
\hline Aca pra & 0,26 & 0,01 & 0,01 & 0,04 & 0 & 0,04 \\
\hline Agr gla & 0 & 0 & 0 & 0 & 0 & 0,04 \\
\hline Asp que & 0,18 & 0 & 0 & 0 & 0 & 0 \\
\hline Asp arg & 0 & 0 & 0 & 0,01 & 0,02 & 0 \\
\hline Bou sti & 0,05 & 0 & 0 & 0 & 0 & 0 \\
\hline Cel ehr & 0,26 & 0,02 & 0,02 & 0,01 & 0,06 & 0 \\
\hline Ces par & 0,25 & 0,01 & 0,01 & 0,03 & 0,01 & 0 \\
\hline Cro hir & 0,02 & 0 & 0 & 0,01 & 0,06 & 0 \\
\hline Cro lac & 0 & 0 & 0 & 0,14 & 0,26 & 0,04 \\
\hline Dol cyn & 0.02 & 0 & 0 & 0,07 & 0,02 & 0,01 \\
\hline Flo ool & 0 & 0 & 0 & 0 & 0,01 & 0,11 \\
\hline Jar ich & 0,01 & 0,06 & 0,14 & 0,01 & 0 & 0,04 \\
\hline Jod rho & 0,06 & 0 & 0 & 0 & 0 & 0 \\
\hline Lit mol & 0 & 0,02 & 0,01 & 0,03 & 0,38 & 0,01 \\
\hline Man pen & 0 & 0 & 0 & 0 & 0,10 & 0 \\
\hline Nas sp. & 0 & 0 & 0 & 0 & 0 & 0,04 \\
\hline Opl hir & 0 & 0 & 0 & 0,08 & 0 & 0 \\
\hline Pas dil & 0,03 & 0,01 & 0,01 & 0 & 0 & 0,02 \\
\hline Por mic & 0,06 & 0 & 0 & 0 & 0 & 0 \\
\hline Pro nig & 0,02 & 0 & 0 & 0 & 0 & 0 \\
\hline Rup ape & 0,02 & 0,01 & 0 & 0 & 0 & 0,02 \\
\hline Sch mar & 0 & 0 & 0 & 0 & 0 & 0,32 \\
\hline Sch fas & 0,13 & 0 & 0,01 & 0 & 0 & 0 \\
\hline Seb com & 0,01 & 0,01 & 0 & 0,63 & 0,13 & 0,01 \\
\hline Set par & 0,15 & 0,01 & 0,03 & 0 & 0 & 0 \\
\hline Zin per & 0 & 0 & 0 & 0 & 0 & 0,04 \\
\hline
\end{tabular}


Las comunidades encontradas fueron:

1) Quebrachales, dominados por Aspidosperma quebracho-blanco, se ubican en zonas bajas e intermedias de la Reserva, hasta los 570 m.s.n.m., correspondiendo a la Provincia Fitogeográfica del Espinal, Distrito Cordubense (LEWIS et al., 1973) y sus áreas ecotonales con el Chaco Serrano. Están asociados a las zonas más bajas de la cuenca, definidos como parches remanentes de los procesos de avance de la agricultura. Esta especie está asociada a Acacia praecox Griseb de tipo arbóreo, Celtis ehrenbergiana (Klotzsch) Liebm., Jodina rhombifolia (Hook. y Arn.) Reissek, Porlieria microphylla (Baill.) Descole, O’Donell y Lourteig, Cestrum parqui L'Hér. y Schinus fasciculatus var. fasciculatus (Griseb.) I.M. Johnst. Se observa una alta cantidad de renovales de quebracho blanco fuera de los parches, sobre área agrícola, lo que indica un buen potencial de recuperación, aunque es necesario evaluar la sobrevida de los mismos. Poseen una riqueza media de 29 especies y un valor medio de diversidad de 2,79. Ubicados en zonas ecotonales entre el Chaco Serrano y el Espinal.

2) Blanquillales, se desarrollan sobre cursos permanentes o temporarios de agua, o en su defecto sobre laderas con exposición sur de modo de captar las lluvias. Es por esto que se supone que la especie Sebastiania commersoniana (Baill.) L.B. Sm. y Downs tiene la capacidad de absorber grandes cantidades de agua. Esta especie se asocia a la mora (Morus alba), al siempre verde (Ligustrum lucidum) y al cafeto (Manihot grahamii). Cubren densamente el suelo, no permitiendo el desarrollo de otras especies y por ello estas comunidades presentan los menores índices de biodiversidad y riqueza. Poseen una riqueza media de 24 especies y un valor medio de diversidad de 1,71. Es común encontrar la especie Croton lachnostachyus Baill., la cual es umbrófila, con coberturas promedio de 14\% (aunque sin diferencias significativas respecto a otras comunidades) (Tabla 2).

3) Mollales, con dominancia de Lithraea molleoides, se desarrollan sobre zonas rocosas. Esta especie y otros árboles asociados como el blanquillo también generan un sotobosque de pobre diversidad florística y baja riqueza. Las poblaciones de molle, asociadas a blanquillos, aumentan su frecuencia gradualmente al ascender topográficamente y al alejarse de las vías de escurrimiento, disminuyendo el blanquillo proporcionalmente. Poseen una riqueza media de 25 especies y un valor medio de diversidad de 2,21. Debido a la alta cobertura del sotobosque también se asocia a los mollales la especie Croton lachnostachyus con coberturas promedio del 20\% (Tabla 2).

4) Espinillales están definidos por Acacia aroma Gillies ex Hook. y Arn. principalmente y A. caven (Molina) Molina en menor medida. En la clasificación supervisada se confunden con los pastizales por la presencia de poáceas como Jarava ichu Ruiz y Pav. var. ichu y Amelichloa brachychaeta (Godr.) Arriaga y Barkworth u otras herbáceas como Aloysia gratissima (Gillies y Hook. ex Hook.) Tronc. var. gratissima, y de acuerdo a la mayor o menor área cubierta de suelo por las Acacias. Los individuos observados no superan los $3 \mathrm{~m}$ de altura. De acuerdo a lo observado suele desarrollarse sobre laderas a sotavento ( $N$, NE y NW), recibiendo menor 
cantidad de agua de lluvia y humedad, por lo que son afectados más frecuentemente por los incendios. Poseen una riqueza media de 26 especies y un valor medio de diversidad de 2,26.

5) Los horcales (u orcales) se disponen, a diferencia de los espinillales, a barlovento (S, SE y E). Los grandes individuos de Schinopsis marginata encontrados y la gran cantidad de renovales encontrados indican que son bosques que sufren menor intensidad y frecuencia de disturbios, y se encuentran en recuperación, a pesar de haber sido diezmados décadas atrás por la extracción de combustible para las caleras de la zona. La chilca (Flourensia oolepis S.F. Blake) es una especie asociada característica. Poseen una riqueza media de 27 especies y un valor medio de diversidad de 2,26.

6) Los pastizales presentan diferente fisonomía de acuerdo a su ubicación relativa respecto a cursos de agua, intensidad de pastoreo, incendios y tipo de suelo. Pueden ser diferenciados en dos clases: a) pastizales con especies saxícolas, dominados por Jarava ichu var. ichu y Amelichloa brachychaeta, y b) pastizales de zonas riparias, dominados por Cynodon dactylon (L.) Pers., la cual es una especie introducida pero posiblemente se trate de la variedad pilosus, asilvestrada. Se encuentran generalmente afectados por alta intensidad de pastoreo. Ambos pueden presentar asociados bosquecillos pequeños de chañar (Geoffroea decorticans (Gillies ex Hook. y Arn.) Burkart). De acuerdo a observaciones personales, cuando los pastizales son afectados por incendios suelen aparecen con abundancia moderada individuos de coco (Zanthoxylum coco Gillies ex Hook. f. y Arn.) los cuales, si sobreviven a las bajas temperaturas invernales pueden formar pequeños bosquecillos. Los pastizales poseen valores medios de riqueza de 26 y 30 especies y 2,25 y 2,28 de diversidad respectivamente.

\section{Clasificación de Unidades de Cobertura de Suelo}

Las clases obtenidas en la clasificación (Figura 1) en base a las observaciones de campo, que respondían a las unidades de 'vegetación' fueron: Bosque Denso (BD), Bosque Abierto (BA), Arbustal-Bosque bajo (A-BB), Pastizal-Arbustal Abierto (P-AA), Pastizal Ripario (PR) y Agricultural $(A C)$.

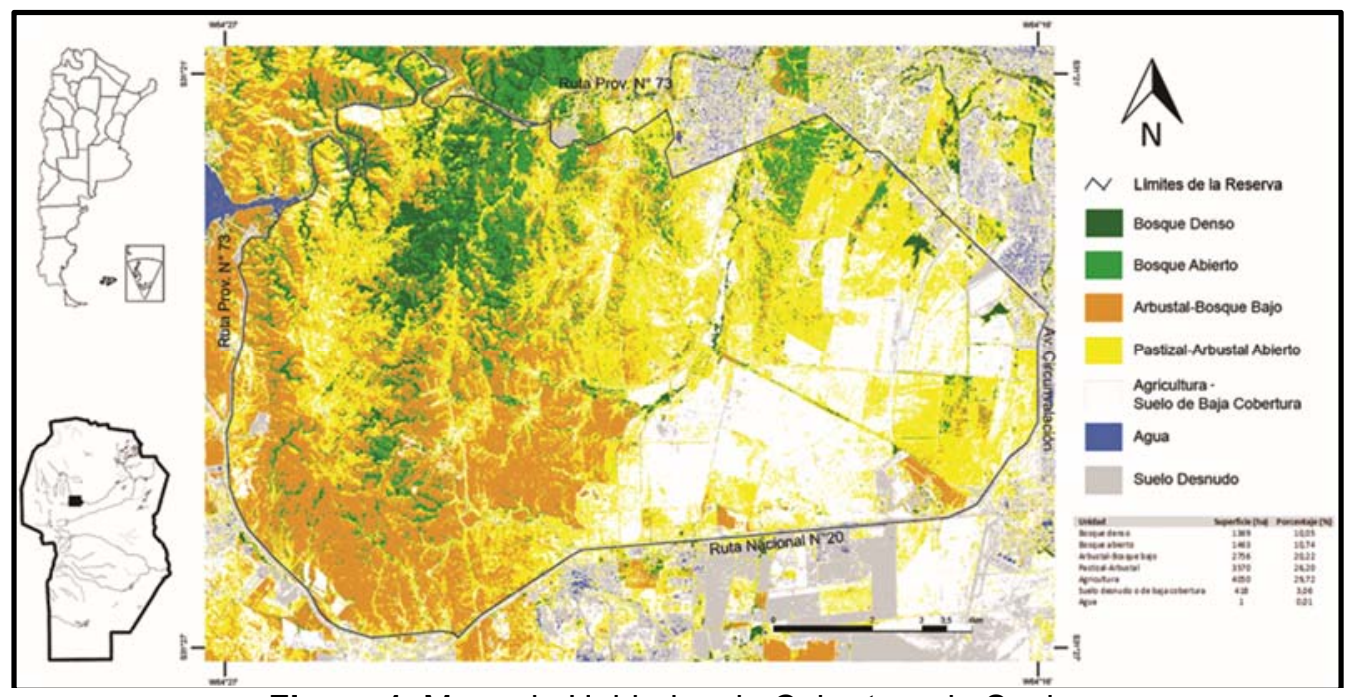

Figura 1: Mapa de Unidades de Cobertura de Suelo. 
1) Bosque Denso: corresponde a los blanquillales asociados a especies exóticas arbóreas, y se observan fundamentalmente sobre las cuencas medias de los cursos principales de agua dentro de la Reserva.

2) Bosque Abierto: incluye las formaciones boscosas de mayor altura, maduros o de mejor estado de conservación correspondientes a las comunidades de quebracho blanco, horco quebracho y molle.

3) Arbustal-Bosque bajo: incluye arbustales o matorrales de diferentes tipos, o zonas de bosque nativo secundario, los cuales se diferencian de la unidad anterior por presentar un estrato arbóreo de baja cobertura o de altura menor, pero con un estrato arbustivo predominante en cobertura y especies. Incluye los espinillales más densos (con coberturas de $A$. aroma y $A$. caven superiores al $15,0 \%)$.

4) Pastizal-Arbustal Abierto: incluye los pastizales nativos, con baja cobertura arbórea (igual o menor al 15,0\%), arbustales de baja cobertura o con arbustos aislados. También incluye los pastizales riparios. Pueden también aparecer incluidos en esta unidad zonas de agricultura o determinados potreros ganaderos, que por su manejo alternan en el tiempo entre herbazales y pastizales nativos y especies exóticas cultivadas o implantadas.

5) Agricultural: corresponde a superficies bajo cultivo o en barbecho, y en zonas altas del área responde a afloramientos rocosos.

En cuanto a las clases de unidad de 'no vegetación', se definieron tres unidades de cobertura del suelo: Agua (Ag), Suelo Desnudo (SD) y Suelo de Baja Cobertura (SBC).

6) Agua: correspondió a cuerpos o cursos de agua naturales o de origen antrópico, tales como diques o canteras inundadas.

7) Suelo desnudo o de muy baja cobertura: áreas correspondientes a caminos, huellas, áreas mineras, zonas de vivienda o áreas asociadas a altas densidades de ganado, como corrales, donde el pastoreo, pisoteo y el bosteo deja reducida a su mínima expresión la cobertura vegetal del suelo.

\section{DISCUSIÓN}

La Reserva Natural Militar Estancia La Calera, con las 230 especies encontradas hasta el momento es un sitio representativo de la ladera oriental de las Sierras de Córdoba. Asimismo, la zona baja de la cuenca constituye un importante reservorio de germoplasma con especies representativas de la ecorregión Espinal Distrito Cordubense, el cual se encuentra en la actualidad seriamente afectada en cuanto a superficie en la Provincia de Córdoba con un $0,1 \%$ remanente (ZAK, 2008, citado por NOY-MEIR et al., 2012). De las 896 especies vasculares citadas por Giorgis et al. (2011) para toda el área correspondiente a las Sierras de Córdoba y San Luis, el $25,7 \%$ fue encontrado en el área de estudio, aunque 17 (7,4\%) especies encontradas no son citadas por estos autores. Si se comparan los porcentajes de exóticas encontradas en la Reserva respecto al total, estas representan el 8,3\%, valor inferior a la proporción de exóticas encontradas 
por Giorgis et al. (2011) las cuales representan el 11\%. La Reserva presenta en la actualidad un menor porcentaje y coberturas de especies exóticas respecto a toda el área de las Sierras de Córdoba y San Luis (GIORGIS et al., 2011) posiblemente como consecuencia de la restricción al ingreso y reducción de disturbios antrópicos, lo que constituye un importante capital frente a la conservación de especies nativas. Estas especies exóticas, se restringen básicamente a zonas riparias afectando la dinámica hídrica de la cuenca. Debido a esto, se considera que el manejo de las especies exóticas debe ser abordado a través de estrategias que tiendan a disminuir progresivamente su participación mediante técnicas silvícolas apropiadas, protegiendo asimismo el suelo de efectos erosivos. Para esto debe considerarse la cobertura afectada, la competencia sobre especies nativas y la proporción de renuevos (COIRINI et al., 2013). De acuerdo a los censos efectuados, las dos especies exóticas de mayor impacto por su abundancia, porte y capacidad de competencia son Morus alba y Ligustrum lucidum, de reconocido alto consumo hídrico (HOYOS et al., 2010)., además de Manihot grahamii como extra regional, siendo el resto poco importante al menos en abundancia, pero a pesar de ello deben ser monitoreadas periódicamente debido a que se observaron gran cantidad de renuevos potencialmente perjudiciales, especialmente de Melia azedarach L. y Robinia pseudoacacia L.

Las especies endémicas constituyen el $17 \%$ del total, lo que hace de esta Reserva un lugar de alto valor de conservación, especialmente sobre cactáceas, muchas de ellas endémicas de las Sierras de Córdoba. Debe considerarse que la proporción en cuanto abundancia de muchas de estas especies endémicas es reducida, por lo que deben identificarse correctamente los nichos donde estas se desarrollan y establecerse áreas de conservación especiales dentro de la Reserva. A pesar de no ser exótica, la especie Sebastiania commersoniana presenta los mayores índices de cobertura en zonas riparias debido a su gran capacidad de expansión en zonas inundables (KOLB et al., 1998). Debe discutirse su rol dentro de este ecosistema y qué efecto potencial puede producir sobre el resto de la flora nativa en estas zonas. Esta especie y las dos exóticas mencionadas (Morus alba y Ligustrum lucidum) provocan una drástica disminución de la diversidad florística en los blanquillales.

Los mollales son, de acuerdo a Giorgis et al. (2011), comunidades boscosas dominantes en el Bosque Serrano. Sin embargo, fue difícil su identificación como comunidad pura en el área de estudio, habiendo encontrado sólo tres sitios, asociados a blanquillales. La identificación de pastizales es de vital importancia debido a que son generalmente el principal foco de incendios de la Reserva, especialmente porque se ubican en zonas donde hay mayor cantidad de tránsito, aledaños a rutas, basurales y zonas urbanas. Estas zonas deben ser manejadas con un adecuado pastoreo a fin de reducir la cantidad de biomasa combustible. Los arbustales, especialmente los espinillales, también presentan elevado riesgo de incendio debido a que la cobertura arbustiva es baja y las pasturas se desarrollan sin mayores restricciones. Los espinillales son originados posiblemente por la influencia de incendios ya que tanto $A$. caven como $A$. aroma tienen gran capacidad de rebrote post fuego (VERZINO et al., 2005). A los fines prácticos, estos podrían ser 
manejados conjuntamente con los pastizales. Los parques boscosos aledaños a los pastizales deben ser protegidos frente a posibles eventos de incendios iniciados en estos últimos, a fin de evitar los incendios de copa.

\section{AGRADECIMIENTOS}

Agradecemos al comando de la II División de Ejército por permitirnos el ingreso a la Reserva Natural Militar La Calera y por acompañarnos en muchas de las entradas a campo. A la Society for Conservation GIS y el ESRI Conservation Program, por la licencia de software de análisis y procesamiento cartográfico ArcGISTM 10 (ESRI尺) y bibliografía sobre el mismo.

\section{REFERENCIAS}

ARANA, M. D.; BIANCO, C. A.. Helechos y licófitas del centro de la Argentina. Rio Cuarto: UNRC, 2011. BRAUN-BLANQUET, J.. Fitosociología: bases para el estudio de las comunidades vegetales. Madrid: Ed. Blume, 1979.

CABIDO, C.; CARRANZA, M. L.; ACOSTA, A.; PÁEZ, S.. Contribución al conocimiento fitosociológico del Bosque Chaqueño Serrano en la provincia de Córdoba, Argentina. Phytocoenología, Buenos Aires, v.19, p.547-566, 1991.

CABRERA, A.. Regiones fitogeográficas argentinas. Buenos Aires: ACME, 1976.

CAPITANELLI, R. G. C.. Geografía Física de la provincia de Córdoba. Buenos Aires: Boldt, 1979.

CANTERO, J. J.; SFRAGULLA, J. A.; NÚÑEZ, C.; BONALUMI, A. A.; MULKO, J.; AMUCHASTEGUI, A.; CHIARINI, F.; BARBOZA, G. E.; ESPINAR, L. A.. Flora de los afloramientos de mármoles y serpentinitas de las Sierras de Córdoba (Argentina). Kurtziana, Córdoba, v.36, n.2, p.11-45, 2011.

COIRINI, R.; KARLIN, M.; BRASSIOLO, M.. Bosque Nativo. Manual de buenas prácticas y propuestas de producción sostenible, ecorregión Espinal. Buenos Aires: Secretaría de Ambiente y Desarrollo Sustentable de la Nación (en prensa), 2013.

DALMASSO, A.; CANDIA, R.; LLERA, J.. La vegetación como indicadora de la contaminación por polvo atmosférico. Multequina, Mendoza, v.6, p.85-91, 1997.

DEMAIO, P.; KARLIN, U.; MEDINA, M.. Árboles del centro de Argentina. Buenos Aires: LOLA, 2002.

DI GREGORIO, A.; JANSEN; L. J.. Land Cover Classification System. Roma: Food and Agriculture Organization of the United Nations (FAO), 2005.

DI RIENZO, J.; CASANOVES, F.; GONZALEZ, L.; TABLADA, M.; ROBLEDO, C.; BALZARINI, M.. Infostat. Software estadístico. Córdoba: Facultad de Ciencias Agropecuarias, Universidad Nacional de Córdoba., 2012.

ETIENNE, M.; PRADO, C.. Descripción de la vegetación mediante la cartografía de ocupación de tierras. Conceptos y Manual de Uso Práctico. Santiago de Chile: Departamento de Producción Animal. Facultad de Ciencias Agrarias, Veterinarias y Forestales, Universidad de Chile, 1982.

GAILLARD DE BENITEZ, C.; PECE, M. G.. Muestreo y técnicas de evaluación de vegetación y fauna. Santiago del Estero: Universidad Nacional de Santiago del Estero, 2011.

GAVIER, G.; BUCHER, E.. Deforestación de las Sierras Chicas de Córdoba (Argentina) en el período 1970-1997. Córdoba: Academia Nacional de Ciencias, 2004.

GIORGIS, M.; CINGOLANI, A. M.; CHIARINI, F.; CHIAPELLA, J.; BARBOZA, G.; ARIZA ESPINAR, L.; MORERO, R.; GURVICH, D. E.; TECCO, P. A.; SUBILS R.; CABIDO, M.. Composición florística del Bosque Chaqueño Serrano de la provincia de Córdoba, Argentina. Kurtziana, Córdoba, v.36, n.1, p.9-43, 2011.

GURVICH, D. E.; ENRICO, L.; CINGOLANI, A. M. Linking plant functional traits with post-fire sprouting vigour in woody species in central Argentina. Austral Ecology, Alice Springs, v.30, p.789-796, 2005. 
HOYOS, L. E.; GAVIER-PIZARRO, G. I.; KUEMMERLE, T.; BUCHER, E. H.; RADELOFF, V. C.; TECCO, P. A.. Invasion of glossy privet (Ligustrum lucidum) and native forest loss in the Sierras Chicas of Córdoba, Argentina. Biological Invasions, v.12, p.3261-3275, 2010.

KIESLING, R.; FERRARI, O.. 100 cactus argentinos. Buenos Aires: Albatros, 2005.

KOLB, R. M.; MEDRI, M. E.; BIANCHINI, E.; PIMENTA, J. A.; GILONI, P. C.; CORREA, G. T.. Anatomia ecológica de Sebastiania commersoniana (Baillon) Smith y Downs (Euphorbiaceae) submetida ao alagamento. Brazilian Journal of Botany, Sao Paulo, v.21, n.3, 1998.

LEWIS, J. P.; COLLANTES, M. B.. El Espinal Periestépico. Ciencia e investigación, Buenos Aires, v.29, p.360-377, 1973.

LUTI, R.; BERTRÁN DE SOLÍS, M. A.; GALERA, M. F.; MÜLLER DE FERREIRA, N.; BERZAL, M.; NORES, M.; HERRERA, M. A.; BARRERA, J. C. Vegetación. En: VÁZQUEZ, J.; MIATELLO, R.; ROQUÉ, M..

Geografía Física de la provincia de Córdoba, Buenos Aires: Boldt, p.297-368, 1979.

MALDONADO, F.; CARVALHO, V.; SOUSA, C.; MARTINELLI, M.; PINHEIRO JUNIOR, O.; FERNANDES, S. F.. Determinación de la longitud de transecta para el relevamiento fisonómico-estructural de la vegetación del semiárido para suministrar datos a las técnicas de percepción remota orbital. Multequina, Mendoza, v.13, p.1-14, 2004

MORELLO, J. H.; ADÁMOLI, J.. Las grandes unidades de vegetación y ambiente del Chaco Argentino. Buenos Aires: INTA, 1968.

MUELLER-DOMBOIS, D.; ELLENBERG, H.. Aims y methods of vegetation ecology. Nova York: John Wiley \& Sons, 1974.

NOY-MEIR, I.; MASCÓ, M.; GURVICH, M. D.; PERAZZOLO, D.; RUÍZ, G.. Estructura y diversidad de dos fragmentos del bosque de Espinal en Córdoba, un ecosistema amenazado. Boletín de la Sociedad Argentina de Botánica, Córdoba, v.47, n.1-2, p.119-133, 2012.

PALACIOS, A.; SPICOGNA, J.; BERNASCONI, J.; BUDINI, I.; RUFINI, S.; FERREYRA, Y.; SALGUERO, E.; DÍAZ ROMERO, D.. Decidirnos a decidir. Córdoba: UNC, 2010.

ROIG, F. A.. El cuadro fitosociológico en el estudio de la vegetación. Contribuciones del Instituto Argentino de Investigaciones de las Zonas Áridas. Deserta, Mendoza, v.4, p.45- 67, 1973.

SÉRCIC, A.; COCUCCI, A.. Flores del centro de Argentina. Córdoba: Academia Nacional de Ciencias, 2010.

SUÁREZ, S.; VISCHI, N.. Caracterización fisonómico estructural de la vegetación serrana (Alpa CorralCórdoba-Argentina). Multequina, Mendoza, v.6, p.21-32, 1997.

TREVISSON, M.; DEMAIO, P.. Cactus de Córdoba y el centro del país. Buenos Aires: LOLA, 2006.

VERZINO, G.; JOSEAU, J.; DORADO, M.; GELLERT, E.; RODRÍGUEZ REARTES, S.; NÓBILE, R.. Impacto de los incendios sobre la diversidad vegetal, Sierras de Córdoba, Argentina. Ecología Aplicada, La Molina, v.4, n.1-2, p.25-34, 2005.

VOLKMANN, L. R.. Enredaderas de las Sierras de Córdoba. Córdoba: Imprenta de La Mancha, 2005.

VOLKMANN, L. R.; VON MÜLLER, N. Trepadoras y apoyantes más comunes de la Sierra Chica. Córdoba: Prosopis Editora, 1999.

WIKUM, D. A.; SHANHOLTZER, G. F.. Application of the Braun-Blanquet Cover-Abundance Scale for vegetation analysis in land development studies. Environmental Management, Nova York, v.2, n.4, p.323$329,1978$.

ZAK, M.; CABIDO, M.. Spatial patterns of the Chaco vegetation of central Argentina: Integration of remote sensing and phytosociology. Applied Vegetation Science, Malden, v.5, p.213-226, 2002. 